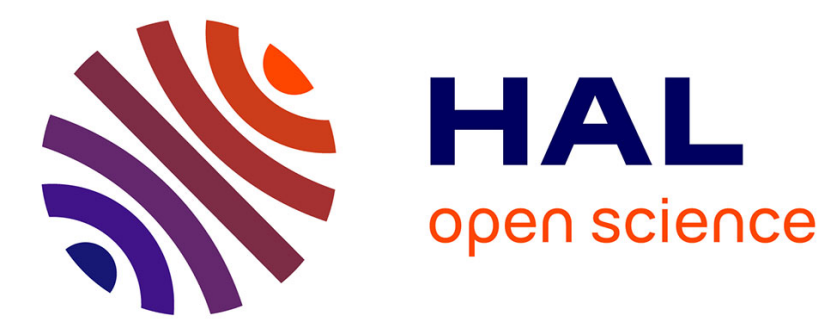

\title{
Portrait du récollet en écrivain au XVIIe siècle
}

Fabienne Henryot

\section{To cite this version:}

Fabienne Henryot. Portrait du récollet en écrivain au XVIIe siècle. Les récollets 1612-2012. En quête d'une identité franciscaine, Jun 2012, Paris, France. pp.219 - 233. hal-01103769

\section{HAL Id: hal-01103769 \\ https://hal.science/hal-01103769}

Submitted on 15 Jan 2015

HAL is a multi-disciplinary open access archive for the deposit and dissemination of scientific research documents, whether they are published or not. The documents may come from teaching and research institutions in France or abroad, or from public or private research centers.
L'archive ouverte pluridisciplinaire HAL, est destinée au dépôt et à la diffusion de documents scientifiques de niveau recherche, publiés ou non, émanant des établissements d'enseignement et de recherche français ou étrangers, des laboratoires publics ou privés. 


\section{Portrait du récollet en écrivain au XVIIe siècle}

Publié dans : C. Galland, F. Guilloux, P. Moracchini (dir.), Les récollets, en quête d'une identité franciscaine,

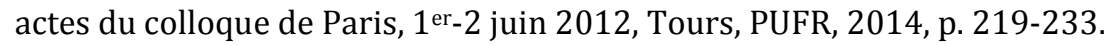

Fabienne Henryot

Bibliothèque cantonale et universitaire de Lausanne LARHRA - UMR 5190 - Équipe RESEA

En 1728, Candide Chalippe, récollet de la province Saint-Denis, publie à Paris une Vie de saint François. Il aurait agi sur une suggestion d'Adrien Baillet qui aurait déploré l'absence, au sein de la vaste famille franciscaine, d'un biographe compétent du fondateur de l'ordre. Le P. Chalippe a alors environ 60 ans $^{1}$. La reconnaissance de son travail va bien au-delà de son ordre, si on en juge par l'appréciation donnée à son ouvrage par Leullier, censeur royal, théologien et approbateur de cet ouvrage : " La vie de saint François est écrite avec beaucoup d'exactitude [...] On trouve par tout une narration fidelle, une Théologie exacte, une Morale pure, le zèle ardent de la foi, des Réflexions sensées et salutaires, des Notes sçavantes et utiles, un stile doux et coulant ».

À ce moment, Chalippe serait, selon nos calculs, le $32^{\mathrm{e}}$ récollet à se faire un nom dans les lettres au sein de la province Saint-Denis. Son cas est particulièrement intéressant car en s'emparant de saint François avec une réelle compétence (l'ouvrage est republié à plusieurs reprises au XIXe siècle ${ }^{2}$ ), il affirme sans détours que le Pauvre d'Assise fut aussi le premier écrivain de l'ordre. Voilà qui peut surprendre dans une famille religieuse longtemps déchirée par la question de la place à accorder aux études, aux savoirs et partant, à l'écriture sous toutes ses formes ${ }^{3}$. Sans compter que les frères mineurs, au cours du XVI siècle, n'ont pas été très actifs dans le domaine de l'écriture, en France tout au moins ${ }^{4}$. À rebours de ces déchirures internes au franciscanisme et de ce constat de médiocrité, Chalippe présente résolument saint François comme l'archétype de l'écrivain religieux de l'âge classique ${ }^{5}$. Saint François, en effet, lors des deux phases de

\footnotetext{
${ }^{1} \mathrm{BnF}, \mathrm{ms}$. fr. 13875, Nécrologe des frères mineurs récollets de la province Saint-Denys depuis sa fondation, fol. 11. LouisFrançois-Candide Chalippe meurt à Paris le 7 janvier 1757, âgé de 88 ans et 74 en religion. Il était doyen de la province de Paris.

2 C. CHALIPPE, La Vie de S. François d'Assise, instituteur de l'Ordre des Frères mineurs, de celui de sainte Claire, et du Tiers ordre de la Pénitence... par le père Candide Challippe... Nouvelle édition, augmentée du panégyrique du saint... et du bref de N. S. P. le Pape Pie VII sur la découverte du corps de S. François, faite à Assise en 1820, Avignon, 1824, 3 vol.; rééd. même adresse 1841 ; rééd. Paris, 1867 ; rééd. même adresse 1874.

${ }^{3}$ Sur cette vaste question, on pourra lire les mises au point d'A. BARTOLI-LANGELI, «I libri dei frati. La cultura scritta dell'ordine dei Minori», Francesco d'Assisii e il primo secolo di storia francescana, Turin, 1997, p. 283-305; C. PAOLAZZI, «I frati minori e i libri : per l'esegesi di 'ad implendum eorum officium' et 'nescientes litteras' », Archivum Franciscanum Historicum, 97, fasc. 1-2, 2004, p. 3-60; R. RUSCONI, «I frati Minori dell'Osservanza in Italia dopo il Concilio di Trento: circolazione di libri e strumenti di formazione intellettuale (sulla base delle biblioteche conventuali e personali) », F. Meyer et L. Viallet (dir.), Identités franciscaines à l'âge des réformes, Clermont-Ferrand, 2005, p. 385-408.

4 M.-M. FRAGONARD, «Les publications des franciscains : une mutation d'identité », Foi, fidélité, amitié en Europe à la période moderne. Mélanges offerts à R. Sauzet, Tours, Publications de l’Université, 1995, t. 1, p. 177-187.

5 Ph. MARTIN, Une religion des livres (1640-1850), Paris, 2003.
} 
rédaction de la Règle, n'écrit qu'après avoir prié ; le texte est dicté par le Christ luimême ; il n'en demande pas moins les approbations officielles auprès du pape ; il est mû par la charité à l'égard de ses frères : autant de traits qui, à partir du début du XVII siècle ${ }^{6}$, caractérisent l'écrivain catholique exemplaire, qui ne porte ses mots sur le papier que sous l'effet de la grâce et au bénéfice gratuit de son prochain.

Chalippe présente donc l'Observance franciscaine comme un mouvement sinon intellectuel, du moins porté aux activités de l'esprit et l'écriture comme un acte de transformation sociale. On se propose ici de comprendre comment s'est tracé cet itinéraire paradoxal des fils de saint François au sein d'un monde que l'écrit tend, au XVII ${ }^{\text {e }}$ siècle, à envahir ${ }^{7}$. Tout commence en 1605 quand est mis au jour le premier écrit avec un label "récollet», dû à la plume de Nathanaël Le Sage, figure emblématique de l'introduction de la réforme récollette en France à partir de $1601^{8}$. Nous interrompons cette trajectoire en 1728, à la parution du livre de Candide Chalippe. Qu'est-ce qu'écrire? Qu'implique cet acte ? Comment les religieux assument et revendiquent-ils cette activité au cœur du monde clérical où la concurrence des plumes est rude, enfin dans un monde où il y a tant à faire?

Les récollets, à la différence d'autres ordres, monastiques ou mendiants, n'ont pas produit de bibliographie raisonnée en vue de faire valoir leur place dans le monde de l'écrit, au contraire des capucins, dominicains, carmes, célestins ou bénédictins qui se dotent, dans la première moitié du XVIII siècle, de Bibliothecae ou de catalogue de scriptores $^{9}$. On objectera que les Scriptores ordinis minorum de Luc Wadding énumèrent un certain nombre d'auteurs récollets ${ }^{10}$; mais il s'agit uniquement de religieux de la première génération, qui ne sont pas désignés récollets mais inclus au sein d'un vaste ensemble franciscain où les obédiences ne sont pas clairement signalées. De même, la Bibliotheca universa francescana du franciscain déchaux espagnol Jean de Saint-Antoine, trop ambitieuse, rassemble dans un même recueil des cordeliers, des récollets, des capucins et des tertiaires réguliers, selon une sélection extrêmement hasardeuse et donc non satisfaisante pour cette étude ${ }^{11}$.

Aussi, pour mesurer l'étendue des productions écrites des récollets, faute de listes toutes établies, nous devons prospecter dans les documents survivants. Si on pose l'hypothèse que c'est la publication qui fait l'écrivain, l'écriture n'étant pas seulement le geste de porter des mots sur le papier, mais un statut reconnu dans l'espace social et savant au cours de l'âge classique ${ }^{12}$, on doit en effet circonscrire les productions récollettes aux seuls textes ayant donné lieu à une impression chez un typographe bénéficiant aussi de réseaux de diffusion plus ou moins étendus. Cela exclut donc deux autres niveaux d'écriture : celui de la simple prise de notes, largement pratiquée par

\footnotetext{
${ }^{6}$ C'est précisément au début du XVIIe siècle que les textes de saint François font l'objet de plusieurs publications sous le titre d'opuscula, ce qui témoigne d'une nouvelle perception des écrits du saint, constituant une véritable œuvre. $B$. $P$. Francisci Assisiatis Opuscula, nunc primum collecta... et commentariis asceticis illustrata per Fr. Lucam Waddingum, Anvers, 1623.

7 H.-J. MARTIN (dir.), Histoire de l'édition française, t. II : Le livre triomphant (1660-1830), Paris, Promodis, 1984.

${ }^{8}$ N. LE SAGE, Traicte de la Reformation de l'ordre du seraphic Père S. François..., Arras, 1605. Sur ce personnage, voir P. MORACCHINI, « Notes sur l'histoire des premiers récollets français », Franciscana, 1, 1999, p. 319-340.

9 Voir la présentation de ce type de sources dans F. HENRYOT, « Les capucins et l'écriture aux XVIIe et XVIIIe siècles d'après la Bibliotheca de Bernard de Bologne », Études franciscaines, n.s., 4, 2011, p. 111-143.

10 L. WADDING, Scriptores ordinis Minorum, Rome, 1650 ; rééd. Rome, 1806.

11 Jean de Saint Antoine, Bibliotheca universa franciscana, sive alumnorum trium ordinum SPN Francisci qui ab ordine seraphico condito, usque ad praesentem diem, Latina, sive alia quavis lingua scripto aliquid consignarunt, Madrid, 17321733, 3 vol. Par rapport au corpus rassemblé pour cette étude, Jean de Saint Antoine ne recense que 52,6\% des auteurs récollets français du XVIIe siècle.

12 A. VIALA, Naissance de l'écrivain. Sociologie de la littérature à l'âge classique, Paris, 1985.
} 
tous les clercs à l'époque moderne dans le cadre de la prédication, et la copie d'ouvrages dans une perspective de dévotion ${ }^{13}$.

Une recherche minutieuse dans les catalogues collectifs, généralistes ou spécialisés des bibliothèques françaises ou étrangères ${ }^{14}$ a permis de mettre au jour 86 auteurs récollets ayant publié un ouvrage ou un simple écrit de circonstance ${ }^{15}$ avant 1728, pour 217 titres, rééditions comprises. C'est assez peu : les capucins, à la même époque, en ont produit 356 dans le même espace ${ }^{16}$. Le cadre retenu est celui d'un espace francophone affectant les contours du Royaume de France à la veille des guerres de Louis XIV (donc sans la province Saint-André au nord). Cet espace est découpé en huit entités provinciales: Saint-Denis (1612), Saint-Bernardin en Provence (1612), l'Immaculée-Conception en Guyenne (1614), Sainte-Marie-Madeleine en Anjou (1619), Saint-Pierre en Bretagne (1620), Saint-François en Dauphiné (1620), Saint-Sacrement en Aquitaine (1635) et Saint-Antoine en Artois (1668). Toutefois, celle de Bretagne n'a donné aucun écrivain.

Après avoir défini la condition de l'auteur chez les récollets d'après les textes juridiques, historiques, sa place dans la construction de la spécificité récollette dès l'introduction de la réforme en France, et sa fonction dans le " dispositif de légitimation de l'action " 17 des religieux, on examinera les données biographiques recueillies pour établir un profil et une carrière caractéristique de l'écrivain dans cet ordre.

\section{Qu'est-ce qu'un auteur?}

Au cours de l'âge classique, le concept d'écrivain quitte définitivement le champ des seules "écritures » administratives pour devenir une qualification sociale. L'auteur est celui qui s'est approprié des normes rédactionnelles édictées en dehors de lui - c'est particulièrement vrai pour les genres religieux que sont la dévotion ${ }^{18}$ et la parénétique ${ }^{19}$ - et a conscience de l'existence d'un public vers lequel il développe des stratégies particulières. Pour cerner cet individu, il faut croiser les sources.

Ce sont d'abord les textes constitutionnels qui, en creux, renseignent sur le statut de l'écrivain récollet. Celui-ci, à vrai dire, n'existe pas à proprement parler. Les statuts des différentes provinces ${ }^{20}$ ne font qu'allusion à l'« auteur » ou à l'« écrivain », hormis les

\footnotetext{
13 F. HENRYOT, «Croire, savoir, se souvenir. Typologie des gestes de l'écrit dans l'univers régulier à l'époque moderne », Cl. Clivaz, J. Meizoz, F. Vallotton (dir.) Lire demain. Des manuscrits anciens à l'ère digitale. Reading Tomorrow. From Ancient Manuscripts to the Digital Area, Lausanne, 2012, p. 541-562.

${ }_{14}$ Recherches dans les catalogues de la Bibliothèque nationale de France, du Catalogue collectif de France (SUDOC compris), de la BNU Strasbourg, de la Bibliothèque franciscaine de Paris, dans le Karlsruhe Virtual Katalog.

15 Nous avons exclu toutefois les innombrables documents de la gestion (factums, ordonnances, lettres circulaires etc.), les constitutions et les ouvrages liturgiques.

16 Chiffre établi à partir de Bernardin de Bologne, Bibliotheca scriptorum ordinis minorum sancti Francisci capuccinorum, Venise, 1747.

17 Nous adoptons ici les problématiques ouvertes par St. VAN DAMME, «Écriture, institution et société. Le travail littéraire dans la Compagnie de Jésus en France (1620-1720) », Revue de synthèse, 120, n 2-3, 1999, p. 261-283, quoiqu'avec des sources très différentes.

18 Ph. MARTIN, op. cit.

19 Parmi une abondante littérature sur la prédication au XVIIe siècle, citons J.-P. LANDRY (dir.), Le temps des beaux sermons, Lyon, 2006 ; I. BRIAN, « Des professionnels de la chaire dans la France de l'âge classique », Revue de synthèse, $133, \mathrm{n}^{\circ} 2,2012$, p. 251-272.

20 Statuts consultés : pour la province Saint-Denis : Statuts pour les frères mineurs récollets de la Province de S. Denys en France, ou de Paris, arrestés au chapitre provincial tenu au Convent de Montargis le cinquième de Juin mil six cens trentedeux, Rouen, 1637 ; Statuts des frères mineurs récolets de la province de S. Denis en France. Reçus et approuvez par le
} 
textes de la province de l'Immaculée-Conception (1629), de Saint-Bernardin (1662) et de Saint-Antoine en Artois $(1686,1716)$, où il est question « Des autheurs des livres » et des " escrivains ", mais sans règlementation concrète de cette activité. À l'inverse de ce qui se passe, par exemple, dans la compagnie de Jésus - ordre concurrent des récollets à bien des égards ${ }^{21}$ - où la fonction de scriptor est mentionnée dès le début du XVII ${ }^{\mathrm{e}}$ siècle aux côtés des missionnaires, prédicateurs et enseignants, on ne constate rien de tel chez les récollets. La question est latéralement abordée au paragraphe consacré à " l'impression des livres ", qui réitère l'exigence du Concile de Trente de ne rien publier anonymement et sans autorisation écrite du Général ou du Provincial.

Deux logiques se distinguent. Dans les provinces d'Artois, de l'Immaculée Conception et de Saint-Denis, ce paragraphe est inséré dans le chapitre concernant «la manière de converser hors du cloître », où il est aussi question de la prédication, des voyages, des quêtes et des confesseurs. L'auteur, même s'il écrit au secret d'une bibliothèque où, plus souvent, de sa cellule sans quitter son couvent, est un "voyageur » à la manière de ses textes, susceptibles de circuler. Dans les provinces de Saint-François et de Saint-Bernardin, et dans la custodie Saint-Nicolas de Lorraine, le sujet est abordé dans une argumentation qui relie, cette fois, la prédication, les études, le fonctionnement de la bibliothèque et le processus de publication d'ouvrages, dans une perspective davantage intellectuelle. Mais dans les deux cas, l'écriture est le pendant de la prédication. Les commentaires de la règle rédigés par des récollets établissent à leur tour nettement ce lien entre mobilité, écriture, savoirs et bibliothèques ${ }^{22}$, ce qui mérite d'être souligné quand on sait combien saint François avait hésité sur la place du livre au sein de son ordre, et l'avait finalement admise, à contrecœur, pour les seuls prédicateurs, brèche dans laquelle les franciscains de toutes robes allaient s'engouffrer.

Toutefois, l'application à l'étude ou la pratique de la prédication ne débouchent pas nécessairement sur l'écriture et encore moins sur la publication. Il faut chercher ailleurs, en dehors de l'édiction des lois qui régissent la vie quotidienne des frères en fonction de leur statut et des tâches qui y sont associées, les traces d'une instrumentalisation de l'écriture en vue de conquérir une place dans le monde. Les annales, chroniques et topographies des provinces fournissent des éléments intéressants. Charles Rapine, dans son Histoire générale de l'origine et progrez des [...] récollects (1631), ne fait encore que de timides allusions aux vertus du livre dans toute entreprise pastorale, en insistant sur l'importance des études conventuelles et la

\footnotetext{
chapitre provincial assemblé à Paris la présente année 1698, Paris, 1698 ; Statuts des frères mineurs récolets de la province de Saint-Denis en France, reçus et approuvez par le Chapitre Provincial assemblé à Paris le 17 octobre 1710, Paris, 1715. Pour la province Saint-Antoine: Statuts des récolets de la province de S. Antoine en Artois, veus, receus et approuvez du consentement de toute la province, par les Vocaux du chapitre provincial célébré au convent de Saint-Omer le 20 d'avril 1686, Saint-Omer, 1686 ; Statuts des FF. mineurs récolets de la province de St Antoine en Artois, revus, reçus et approuvez du consentement de toute la Province, par les Vocaux du chapitre Provincial célébré au Couvent d'Arras le 21 de juin 1716, Arras, 1716. Pour la province de Saint-Bernardin : Les Statuts de la province de S. Bernardin en France des Frères Mineurs de la plus étroite observance dits Recolez, Avignon, 1662. Pour la province de l'ImmaculéeConception: Statuta fratrum minorum recollectorum provinciae immaculatae conceptionis B. Mariae Virginis in Aquitania, Limoges, 1629. Pour la province Saint-François : Constitutiones et statuta Fratrum Minorum Recollectorum, provinciae S. Francisci in Gallia, Lyon, 1630 ; Pour la custodie Saint-Nicolas de Lorraine : Statuts des récollets de la custodie de Saint-Nicolas en Lorraine, Luxembourg, 1702.

${ }^{21}$ Par exemple dans le cadre des missions : C. GALLAND, Pour la gloire de Dieu et du Roi. Les récollets en NouvelleFrance aux XVIIe et XVIIIe siècles, Paris, 2012.

22 S. PICOT, La règle de saint François enrichie du texte de l'Évangile sur lequel elle a été fondée, Chambéry-Marseille, 1704 ; I. COTTART, La Règle et le Testament de Notre Père Séraphique Saint François... avec les sentimens du mesme Saint François sur l'étude des sciences humaines pour le salut du prochain..., Paris, 1661.
} 
formation des jeunes frères - à rebours d'une faction anti-intellectuelle encore vivace dans l'Observance au début du XVIIe siècle 23 .

C'est Placide Gallemant qui, en 1649, dans sa description raisonnée de la province Saint-Denis, esquisse un tableau plus net de l'écrivain récollet et de son outillage intellectuel et documentaire ${ }^{24}$. Après avoir énuméré les religieux remarquables par leur doctrine, tels Florent Boulanger ou Didace David, il consacre un chapitre aux écrivains d'une province qui a juste un tiers de siècle. Le palmarès n'est déjà pas négligeable, avec treize écrivains pour cette jeune entité25. La plupart d'entre eux, à part Florent Boulanger, ne figurent pas parallèlement dans le chapitre consacré aux religieux savants. L'écriture n'est donc pas liée à la science, mais plutôt au soin des âmes, à en juger par les types d'ouvrages énumérés par Gallemant : sermons pour Ignace Le Gault ou Jean-Marie l'Escrivain, encadrement des clarisses pour Florent Boulanger, glose de la règle à l'usage des frères pour Polycarpe Du Fay ou François Pothron, ouvrages de dévotion pour Charles Rapine, Jean Rabasse, Marc Le Tellier, Ildephonse Cottart, écrits le plus souvent, souligne Gallemant, en langue vulgaire, ce qui confirme leur destination au plus grand nombre. L'écriture est valorisée comme un moyen d'action au cœur du monde - ou du couvent - aux effets d'autant plus bénéfiques qu'ils peuvent durer longtemps.

C'est encore la stratégie d'Hyacinthe Le Febvre, provincial de Saint-Denis, lorsqu'il publie moins de vingt ans plus tard son Histoire chronologique de la province des Récollets de Paris dans un but clairement publicitaire à l'égard des laïcs et des religieux de son temps ${ }^{26}$. Sa liste des " écrivains de la province », à la différence de celle de Placide Gallemant, est assortie d'un commentaire qui fait de l'écriture une activité non pas accidentelle, affaire de quelques religieux d'exception ou ambitieux, mais bien au cœur de la vocation récollette.

\begin{abstract}
Si un homme Evangélique travaillant à son salut, doit encore procurer celuy du prochain en trois manières, sçavoir par le bon exemple de sa sainte vie, par ses ferventes prédications, \& par ses doctes écrits, ainsi qu'on fait les Apostres \& les Pères de l'Eglise; un frère mineur véritable enfant de saint François, par la profession de sa règle toute Evangélique, ne doit pas seulement se sanctifier, mais il doit encore s'employer au salut des Ames, en ces trois manières, comme en a usé nostre Séraphique Père saint François [...]. C'a esté dans la veuë de cette obligation, que plusieurs sçavans Religieux de cette province, ne se sont pas contentez de s'y sanctifier par l'exacte observance d'une vie Reguliere, \& par leurs continuelles Prédications, mais encore par les doctes écrits qu'ils ont laissez à la postérité, afin d'enseigner les véritez du Ciel, aussi bien après leur mort, à ceux qui viendront après eux, que pendant leur vie à ceux qui leur estoient contemporains ${ }^{27}$.
\end{abstract}

La liste compte cette fois 21 écrivains, y compris lui-même. Mais il en a omis plusieurs : Urbain Quillot et son sermon sur la Vierge Marie (Verdun, 1671), Protais Henriet et son Harmonia Evangelica (Paris, 1660), Charles Jouye et son recueil de prières sur la Passion (Rouen, 1645)... La réputation semble avoir davantage guidé la rédaction de cette liste qu'une véritable investigation. Mais là n'est pas l'important. Hyacinthe Le Febvre présente ces 21 religieux comme de parfaits sectateurs de saint François, puisqu'ils ont pris la plume pour annoncer l'Évangile, convertir les hérétiques

${ }^{23}$ F. HENRYOT, Livres et lecteurs dans les couvents mendiants (Lorraine, XVIe-XVIIIe siècles), Genève, 2013, p. 51-60 et 111-115.

24 P. GALLEMANT, Provincia sancti Dionysii fratrum minorum recollectorum in Gallia a venerando Patre Placido Gallemant eiusdem Provinciae Diffinitore, Châlons, 1649.

25 Ibid., p. 48-53.

${ }^{26}$ H. LE FEBVRE, Histoire chronologique de la province des récollets de Paris, sous le titre de saint Denys en France, depuis 1612 qu'elle fut érigée jusqu'en l'année 1676, Paris, 1677.

27 Ibid., p. 111. 
ou catéchiser les fidèles. Une entreprise du même genre est menée dans la province de Saint-Bernardin par Césaire Cambin, archiviste et chroniqueur de sa province, qui produit les notices biographiques des religieux exemplaires ${ }^{28}$. On y trouve, en bonne place, les trois écrivains que la province a déjà vu naître. Antoine de Saint-Michel y est présenté comme un fervent lecteur et comme un auteur prolifique, puisque dix de ses ouvrages ont été publiés et que depuis sa mort survenue en 1650, neuf autres attendent l'impression ${ }^{29}$. Bonaventure Breugne, lui, écrit son manuel sur la prière pour les âmes du purgatoire suite à une grâce reçue et meurt en rédigeant un commentaire de la règle de saint François ${ }^{30}$. Enfin, Cambin établit un lien de entre les prédications parisiennes de Fortuné du Virail et l'écriture, sitôt descendu de chaire, de son traité sur l'Immaculée Conception qu'il venait de défendre ardemment en public ${ }^{31}$. Cambin veut voir dans ces trois personnages l'incarnation de l'idéal récollet, où l'écriture est à la fois sanctification de soi-même, discipline intérieure et charité à l'égard d'autrui.

Ces différents historiographes et topographes récollets énoncent une définition de l'écrivain qui procède d'une relecture historique du franciscanisme. Reste la réalité, que nous pouvons déduire d'un certain nombre d'éléments démographiques et sociologiques relatifs à la carrière des écrivains du corpus.

\section{Un profil professionnel}

Tableau 1 : Répartition des auteurs et des publications par province

\begin{tabular}{|l|c|c|c|c|c|}
\hline Province & $\begin{array}{c}\text { Nb } \\
\text { auteurs }\end{array}$ & $\begin{array}{c}\text { Nb titres (rééd. } \\
\text { comprises) }\end{array}$ & $\begin{array}{c}\text { Rapport } \\
\text { titres/auteur } \\
\text { rééd. comprises) }\end{array}$ & $\begin{array}{c}\text { Nb titres (rééd. } \\
\text { non comprises) }\end{array}$ & $\begin{array}{c}\text { Rapport } \\
\text { titres/auteur } \\
\text { rééd. non } \\
\text { comprises) }\end{array}$ \\
\hline $\begin{array}{l}\text { Immaculée- } \\
\text { Conception }\end{array}$ & 13 & 38 & 2,9 & 30 & 2,3 \\
\hline $\begin{array}{l}\text { Saint- } \\
\text { Bernardin }\end{array}$ & 10 & 26 & 2,6 & 22 & 2,2 \\
\hline Saint-Denis & 32 & 84 & 2,6 & 21 & 2,2 \\
\hline $\begin{array}{l}\text { Saint- } \\
\text { Antoine }\end{array}$ & 1 & 2 & 2 & 18 & 4,5 \\
\hline $\begin{array}{l}\text { Sainte-Marie- } \\
\text { Madeleine }\end{array}$ & 4 & 21 & 5,25 & 24 & 2,4 \\
\hline $\begin{array}{l}\text { Saint- } \\
\text { François }\end{array}$ & 10 & 24 & 2,4 & 12 & 1,7 \\
\hline $\begin{array}{l}\text { Saint- } \\
\text { Sacrement }\end{array}$ & 7 & 13 & 1,8 & 9 & 1 \\
\hline Indéterminée & 9 & 9 & 1 & $\mathbf{1 8 3}$ & \\
\hline Total & $\mathbf{8 6}$ & $\mathbf{2 1 7}$ & $\mathbf{2 , 5}$ & $\mathbf{2 , 1}$ \\
\hline
\end{tabular}

L'écrivain récollet présente des caractéristiques particulières, en termes de carrière, d'implantation géographique et de compétences, caractéristiques qui émergent d'une comparaison entre les religieux de notre corpus.

\footnotetext{
${ }_{28}$ B.M. Avignon, ms. 1444-1447, La chronique des frères mineurs recollects de la province de St Bernardin en France, par le P. Césaire Cambin, 1676.

${ }^{29}$ Ibid., t. II, p. 341-354.

30 Ibid., t. II, p. 531-540.

31 Ibid., t. II, p. 541-545.
} 
Il faut d'emblée souligner la participation inégale des différentes provinces à cette activité. Près de $40 \%$ des écrivains évoluent dans la vaste province Saint-Denis. Le statut de Paris comme capitale culturelle et religieuse, où s'est forgée la condition de l'écrivain, pourrait donner à penser que la ville est le lieu par excellence où exerce l'auteur récollet. Mais au contraire, d'autres provinces dotées de capitales, telle la province Saint-François dont le cœur bat à Lyon, n'a pas vu beaucoup de religieux prendre la plume (12\% du corpus). La province de L'Immaculée-Conception, polycentrée sur Bordeaux, Poitiers, Saintes et Périgueux, n'a guère été plus active $(14,5 \%)$, ni celle de Saint-Bernardin (9,6\%), malgré la présence de pôles urbains comme Avignon et Marseille. Dans ces différentes provinces, la prolixité des auteurs est assez stable, si l'on considère le critère du nombre de titres par auteur : autour de 2,2 (sans les rééditions), valeur un peu supérieure dans la province de Saint-Bernardin grâce la plume infatigable d'Antoine de Saint-Michel (10 titres) et de Séraphin Picot (5 titres), et dans la province de Sainte-Marie-Madeleine, du fait de Pascal Rapine de Sainte-Marie, auteur d'une histoire apologétique du christianisme en sept périodes formant chacune un volume, et de divers recueils de sermons. En revanche, pour les écrivains de la province du SaintSacrement, l'écriture semble une activité plus décousue et accidentelle et aucun religieux ne s'impose comme un professionnel de l'écriture, hormis Bruno Chassaing, devenu pénitencier à Saint-Jean-de-Latran sous les pontificats de Grégoire XV et d'Urbain VIII.

Ces différents espaces partagent une même conception de l'écrivain, à en juger par le profil constant que présentent ces religieux en tous lieux. Dans la province SaintDenis, l'âge moyen à la première publication est de 47 ans et 2 mois $^{32}$. La plupart des religieux qui remettent un manuscrit pour publication ont entre 42 et 52 ans, tel Eloy Hardouin de Saint-Jacques, qui a 43 ans lorsque paraît son Empire de Jésus Christ souffrant dans les cœurs (Paris, 1655). Cet âge implique, chez nombre de religieux, une pratique aguerrie de l'écriture pastorale (prédication notamment) et conventuelle (factums, lettres circulaires, correspondances etc.). Ainsi, Urbain Quillot, lorsqu'il publie son Sermon des grâces que la glorieuse vierge Marie a faites à Verdun et des secours qu'elle lui a donnés, particulièrement contre les hérétiques (Verdun, 1671) à l'âge de 50 ans, a précédemment été gardien du couvent de Saint-Germain en Laye (1654-1656), puis du couvent de Paris (1660-1662), élu définiteur au chapitre de 1663, enfin prédicateur à la cathédrale de Verdun ${ }^{33}$. Il a donc une expérience de l'autorité et maîtrise les codes formels des différentes formes d'écriture pratiquées dans les couvents de l'ordre.

Ce chiffre moyen masque cependant de grandes disparités. En-deçà, on trouve plusieurs religieux de la première génération, tel Charles Rapine, qui a 27 ans quand commence la diffusion commerciale de son Discours de la vie, mort et miracles de $S$. Memie (Châlons, 1625). Au-delà, quelques religieux sont assez âgés quand commence leur carrière d'écrivain. C'est le cas de Vincent Moret, âgé de 66 ans lorsqu'il confie à un imprimeur son manuscrit de La simplicité chrétienne générale (Paris, 1656). D’une manière générale, et même si cette tendance souffre, à nouveau, bien des exceptions, il semblerait que les écrivains les plus jeunes appartiennent aux deux premières générations de religieux, celles de la réforme, des combats pour l'observance et sa diffusion à travers le Royaume. Ignace Le Gault, qui a 39 ans à la parution de son

\footnotetext{
32 Chiffre établi à partir des éléments biographiques livrés par le Nécrologe des frères mineurs récollets de la province Saint-Denys depuis sa fondation, op. cit.

33 H. LE FEBVRE, op. cit., p. 48, 78-79, 110.
} 
premier ouvrage, appartient à cette génération. À l'inverse, à la fin du siècle, tandis que s'est mis en place un "modèle » de carrière, la publication semble réservée à des religieux ayant déjà donné les preuves de leur capacité et leur autorité. Hyacinthe Le Febvre en est l'exemple le plus caractéristique : il a 58 ans lorsqu'il met sous la presse le premier d'une longue série d'ouvrages, son Traité du Jugement dernier (Paris, 1671). Six autres suivront jusqu'à son décès le 11 juin 1694 au couvent de Saint-Denis.

Hyacinthe Le Febvre est aussi exemplaire en ce qu'il présente, en 1671, le profiltype du religieux écrivain de la seconde moitié du siècle. Il se présente au seuil de chacun de ses ouvrages comme provincial, père de province ou commissaire. L'écriture apparaît ainsi comme inévitablement liée à une autre tâche dans l'ordre, qui justifie le recours à la plume et garantit au public la qualité de l'écriture et l'orthodoxie du propos. L'énonciation de l'auteur par lui-même est un paramètre intéressant à considérer dans la construction de la figure de l'écrivain récollet. Les pages de titre révèlent en effet la manière dont les religieux souhaitent être considérés à la fois par leurs pairs et par le public. D'abord, l'anonymat est rare, conformément aux prescriptions faites aux auteurs dans les statuts provinciaux. Marc Le Tellier prend ce parti lorsqu'il publie sa Règle des âmes dévotes en 1627, ainsi qu'Alexandre Pocquelin dans son Sommaire de la vie et mort du Bienheureux Père Pierre d'Alcantara (1622) ou encore Zacharie de Vitré dans ses vers dévots sur la Passion (1659) mais ils sont les seuls. Ce refus de l'anonymat est intéressant à soulever, comparé aux pratiques jésuites qui, au même moment, consistent pour les auteurs dans plus de $40 \%$ des cas à disparaître derrière l'œuvre et l'intention pastorale ${ }^{34}$. Il révèle la volonté, pour les récollets, d'attirer l'attention sur la participation de leur ordre à la construction d'un discours ecclésiastique, et d'établir une réputation apostolique. L'écriture est un instrument de cette stratégie à laquelle chaque religieux apporte sa contribution.

Les auteurs profitent donc de l'espace de la page de titre et de ses règles formelles pour établir leur légitimité à écrire sur tel ou tel sujet. S'ils restent nombreux à restreindre la présentation à la seule mention de "récollet», par souci d'humilité, tel Gabriel Sagard, missionnaire en Nouvelle-France ${ }^{35}$ ou Gabriel Barrier, auteur d'une vie de saint Pierre d'Alcantara36, les autres énumèrent à l'envi leurs titres et qualités.

Tableau 2 : Qualité des auteurs énoncée sur la page de titre

\begin{tabular}{|l|l|}
\hline Qualité énoncée sur la page de titre & Nombre d'écrivains à la revendiquer \\
\hline « récollet» (sans autre précision) & 19 \\
\hline « provincial », « custode », « définiteur» & 13 \\
\hline «prédicateur» & 21 \\
\hline «gardien » & 16 \\
\hline « lecteur » ou « professeur» & 11 \\
\hline « missionnaire » & 2 \\
\hline « maistre des novices» & 1 \\
\hline
\end{tabular}

Remarque : un même religieux peut revendiquer plusieurs fonctions sur une même page de titre

\footnotetext{
${ }^{34} \mathrm{Ph}$. MARTIN, «L'auteur de piété est-il un anonyme ? », F. Gugelot, F. Preyat et C. Vanderpelen (dir.), La croix et la bannière. L'écrivain catholique en francophonie (XVIIe-XXIe siècles), Bruxelles, 2007, p. 75-86.

${ }^{35}$ Le Grand Voyage du Pays des Hurons, situé en l'Amérique vers la Mer douce, ès derniers confins de la Nouvelle France, dite Canada, avec un Dictionnaire de la langue huronne par Frère Gabriel Sagard Théodat, Recollet de S. François, de la province de S. Denys en France, Paris, 1632.

${ }^{36}$ L'histoire de la vie de St-Pierre d'Alcantara, fondateur des pères Dechaux, ou recolez de la province de St Joseph en Espagne... composée par le R.P. Gabriel Barrier, récolé, Tulle, 1674.
} 
L'écriture procède de trois logiques. Elle est, d'abord, un manifeste d'autorité, en représentant une étape dans une carrière, une preuve de polyvalence et l'aboutissement d'une trajectoire dans les plus hautes charges provinciales. Que ce soit à l'échelle d'une communauté ou à celle d'une province, la capacité à faire observer la règle et à réduire les dissensions confère aux religieux une aura qui rejaillit sur le prestige intellectuel. Simon Chevalier, de la province de l'Immaculée-Conception, auteur d'Exercices spirituels en 1632, se présente comme gardien de Tulle; Elzéar Larcher, de la province SaintDenis, exaltant les origines du franciscanisme dans Le Sacré mont d'Olivet (1614), se dit gardien de La Flèche. Être provincial, custode ou définiteur est une autre qualité distinctive : Victorin Poulihot (provincial de l'Immaculée-Conception), Séraphin Picot (provincial de Saint-Bernardin) ou Victorin Tarneau (provincial du Saint-Sacrement) sont de ces religieux qui, arrivés au sommet de leur carrière, restent au milieu de la mêlée en écrivant sur l'observance ou la controverse religieuse.

L'écriture est, ensuite, le revers de la prédication. Parce que celle-ci fait, ellemême, l'objet d'une autorisation (du provincial et de l'évêque du lieu), la qualité de prédicateur trace les contours d'une élite ecclésiastique. Cette fonction est revendiquée bien sûr au seuil de recueils de sermons, comme le fait Constance Rounat, auteur d'homélies et de panégyriques pour les différents temps de l'année qui ont connu un indéniable succès à l'époque moderne. Mais elle justifie aussi toutes les autres entreprises d'écriture, telle de Siméon Mallevaud, de la province de l'ImmaculéeConception, lorsqu'il publie ses Annales Calveriennes, ou l'histoire chronologique de toutes les maisons de la Congregation de N.-D. de Calvaire, ordre de S. Benoist (Angers, 1671). Le lien entre prédication et écriture, déjà observé dans les statuts, est ainsi réaffirmé et vécu ; du reste, la complémentarité de l'oral et de l'écrit est évidente. Le chancelier de l'université de Paris, Loisel, examinant L'adoration perpétuelle du tressaint-sacrement d'Archange Enguerrand, fait observer à propos de l'auteur: «[sa] langue comme une plume dans la prédication de l'Evangile, et la plume comme une langue dans cette production de piété, se trouveront également capables d'imprimer dans les âmes de grands sentimens de respect pour tous les Mystères ${ }^{37}$. » L'écriture et la publication suivent naturellement le discours verbal, et le travail du typographe sur le papier grâce à la presse se double d'un effet identique sur le lecteur, en lui imprimant de saintes pensées.

L'écriture, enfin, se veut la manifestation d'une compétence, déjà prouvée par l'obtention d'une charge de lecteur et par l'enseignement. Zacharie Laselve, religieux de la province du Saint-Sacrement, publie en 1696 un recueil de sermons en quatre épais volumes, l'Annus apostolicus en se présentant comme lecteur en théologie.

En se présentant lui-même au frontispice de son livre, le religieux écrivain revendique son appartenance à l'élite de son ordre. Lorsqu'il se montre trop discret sur ses compétences, ou trop évasif, les approbateurs se chargent de préciser et compléter le curriculum vitae de l'auteur. Tandis que Jacques d'Embrun, de la province SaintFrançois, se borne à signaler qu'il est « recollect » sur la page de titre de ses Maximes et oracles de Jésus Christ (1635), les approbateurs ajoutent qu'il est prédicateur et confesseur des clarisses de Montbrison. Ceux de Fortuné du Virail, de la province Saint-

\footnotetext{
37 A. ENGUERRAND, L'adoration perpétuelle du tres-saint sacrement qui est de faire réparation d'honneur \& amende honorable à Jésus-Christ sur les Autels, pour les injures qui luy sont faites par les Infidèles, les Hérétiques et les Pecheurs dans ce Mystère adorable, $3^{\mathrm{e}}$ éd., Paris, 1698, approbation (1er oct. 1672). Sur cet auteur et ses liens avec les mystiques du XVIIe siècle, voir A. DERVILLE, «Un récollet français méconnu : Archange Enguerrand », Archivum Franciscanum Historicum, 90, fasc. 1-2, 1997, p. 177-203.
} 
Bernardin, camouflé derrière le simple qualificatif de "religieux recollect» au seuil de son Amour de tous les siècles envers l'immaculée Conception de la mere de Dieu (Paris, 1655), mettent au jour sa fonction de prédicateur et de custode. Ces éléments sont donc essentiels dans la construction de la figure de l'auteur, tout à la fois homme de terrain, professionnel de l'orthodoxie et investi d'une autorité juridique sur toute une province.

Enfin, selon une tradition éditoriale déjà ancienne, l'auteur peut être désigné écrivain par ses confrères, par le biais d'hommages versifiés placés au seuil des ouvrages. Bonaventure Breugne, de la province Saint-Bernardin, a orné son manuel intitulé Le commerce des vivants fait en faveur des âmes du purgatoire (1658) d'une de ces pièces, signée F.Z.D.V.R., abréviation qui cache probablement Frère Zacharie de Vitré récollet. Le poète affirme : «Que tu fais un riche métier / Autheur sçavant \& charitable / Lorsque d'un Commerce admirable / Tu t'es rendu le Courratier [courtier] » et établit un parallèle rhétorique entre Christophe Colomb ouvrant la voie au commerce avec le Nouveau Monde et le frère Bonaventure montrant la voie qui relie le monde des vivants et celui des morts et le moyen de négocier leur salut. Ces hommages caractérisent également une autre catégorie d'« auteurs »: les traducteurs. Charles Jouye s'empare ainsi, dans les années 1610, des opuscules ascétiques de Barthélemy Cambi de Salutio, alors disponibles seulement en italien. On ne dira jamais assez le succès phénoménal de cet auteur en France, aux Pays-Bas et jusqu'en Angleterre, succès que n'a égalé aucun auteur dévot français au même moment ${ }^{38}$. Charles Jouye traduit d'abord en français les Sept trompettes spirituelles pour réveiller les pécheurs en 1616, texte qui connaîtra 27 éditions jusqu'à la fin du XIX ${ }^{\mathrm{e}}$ siècle. Il s'attelle ensuite à l'Amoureux de Jésus, publié à Paris en 1618. Les Sept trompettes, annonce la page de titre, sont « traduittes de l'Italien en François » mais aussi " amplifiées », ce qui suggère une réécriture du texte original. À ce titre, Jouye pourrait être considéré comme co-auteur de cet ouvrage dans sa version française. C'est en tous cas ainsi qu'il a été perçu par ses contemporains. Trois de ses confrères lui rendent hommage au seuil de l'ouvrage - et non à Salutio : Laurent de Moulins, Jacques Le Bigot gardien de La Flèche, Macaire de Morennes. Ce dernier écrit : " Jouye ayant cent et cent fois / En vain crié à haute voix / Contre les péchez que vous faittes / Et que vous y tombiez tousiours / A esté vous voyant si sourds / contraint de sonner ses trompettes ». Ces vers tendent à affirmer que Jouye est finalement l'auteur de ce recueil.

Ces hommages littéraires, au même titre que les précisions apportées par les approbations sur le statut de l'auteur, mettent en évidence un cercle étroit de religieux qui définit, au sein de chaque province, un "collège » restreint de personnes qui se désignent les unes les autres comme porte-parole des ambitions pastorales ou intellectuelles de l'ordre. Le positionnement de l'auteur s'en trouve d'autant mieux affirmé et explicite dans le monde des plumes. Il n'est qu'à rapprocher l'énumération des noms des approbateurs avec celle des auteurs, pour constater qu'un auteur sera, un jour, approbateur de l'écrit d'un de ses confrères, et qu'un approbateur peut, un jour ou l'autre, devenir écrivain. Cette situation n'a rien pour surprendre, étant donné que les auteurs revendiquaient justement les compétences qui étaient aussi celles des approbateurs, notamment la charge de lecteur en théologie, philosophie ou Écriture sainte. Nous avons donc affaire à une élite qui se pressent elle-même comme une élite, étant donné l'étroitesse du cercle de religieux concernés, qui se côtoient par ailleurs

\footnotetext{
38 J. HEERINCKX, « Barthélemy Cambi de Salutio », Dictionnaire de spiritualité ascétique et mystique, t. 1, Paris, 1953,
} col. 1264-1266. 
régulièrement dans les chapitres et les définitoires, et dont la mobilité accroît encore la réputation.

Il faut d'abord rappeler que le système des approbations est réservé aux ouvrages que l'on veut imprimer et diffuser par le biais de la librairie. Il s'agit d'un processus de contrôle de l'orthodoxie et de limitation des publications à certains champs. L'auteur doit faire connaître son intention de publication au définitoire, qui le soumet à deux experts désignés soit pour l'occasion (province Saint-Denis par exemple), soit nommés pour une période déterminée par le chapitre provincial (province SaintBernardin). C'est sur l'avis de ces religieux que le provincial délivre ou non l'approbation. Ces experts, des théologiens «doctes \& irréprochables», doivent «s'acquitter de leur office avec beaucoup de zèle et d'intégrité, sans acception de personnes ${ }^{39}$. Du côté des auteurs, nul ne peut se soustraire à cette obligation, quand bien même il serait lui-même provincial. Lorsqu'il publie ses Sermons sur les Évangiles de tous les jours du caresme en 1684, Hyacinthe Le Febvre, quoique provincial des récollets de Saint-Denis, ne peut se passer de cette approbation ; il confie donc son manuscrit à Eustache Maupassant, lecteur en théologie et définiteur, et Olivier Voysimbert, " premier Père des Provinces de Paris et d'Artois, et custode de celle de Paris », pour se faire délivrer ces autorisations.

Ce cas montre bien le fonctionnement très mécanique de ce processus. Approbateurs et écrivains appartiennent aux mêmes strates de la province et le service peut être, à quelques années de distance, mutuel. Ainsi, en 1638, Placide Gallemant alors définiteur et lecteur en théologie, a délivré un avis favorable pour le manuscrit du Martyrologium franciscanum d'Artus du Monstier. En 1653, c'est au tour d'Artus du Monstier, d'examiner La vie du venerable prestre de J.C. M. Jacques Gallemant de Placide Gallemant, et de l'approuver. En 1661, Placide Gallemant est nommé pour la lecture de la Neustria pia d'Artus du Monstier. Au total, parmi les 43 approbateurs recensés dans la province Saint-Denis au cours du XVIIe siècle, 17 avaient déjà été, ou allaient être, écrivains eux-mêmes. Ignace Le Gault a été le plus sollicité, en examinant successivement les manuscrits de l'Histoire générale... des recollectz de Charles Rapine en 1630, puis le Bonaventura selectus seu regularis disciplinae et perfectionis praxis de François Pothron, le Grand Voyage du pays des Hurons de Gabriel Sagard et l'Exposition paraphrastique de l'Épistre de l'apostre S. Paul aux Hébreux de Charles Rapine en 1632 ; en 1639, le Psaltes purpuratus Jesus Christus et La vraye reigle du cordon mystique de Jésus \& de S. François de Charles Rapine, qui donne décidément beaucoup de travail aux approbateurs; enfin, en 1648, le Provincia Sancti Dionysii fratrum minorum recollectorum in Gallia de Placide Gallemant. Dans l'intervalle, il a lui-même publié deux recueils de sermons et un traité de controverse contre le pasteur Ferry de Metz, durant son gardiennat dans cette ville.

Ce contrôle des écrivains les uns par les autres a pu favoriser une certaine uniformisation des pratiques d'écriture, des sujets, de la rhétorique, du style et des ambitions pastorales. Jean-Damascène Le Bret, prédicateur réputé et bibliothécaire du couvent de Paris, eut à expertiser les manuscrits d'Archange Enguerrant, d'Eugène Roger, d'Artus du Monstier et de Hyacinthe Le Febvre, soit des religieux de deux générations différentes, celle des années 1660 et celle des années 1690, avant de s'éteindre lui-même sans avoir eu le temps de publier ses propres textes; sa province s'en est chargée, de manière posthume en 1698-1699, à travers huit épais volumes.

${ }^{39}$ Statuts de la custodie de Saint-Nicolas de Lorraine..., op. cit., p. 115. 
Le cercle des approbateurs et des auteurs s'est resserré, au cours du XVIIe siècle, dans les limites provinciales, alors qu'au début du siècle, il n'était pas rare de voir un religieux d'une province obtenir ses autorisations d'un religieux d'une autre province. Ainsi, parmi les approbateurs de la Généalogie de l'âme fidelle d'Hilaire Montlouis ${ }^{40}$, prédicateur de la province de l'Immaculée-Conception, ou trouve Isidore de Thiers, custode de la province de Lyon. Au milieu du siècle encore, Le manuel du bon religieux d'Ildephonse Cottart $^{41}$ (religieux de la province Saint-Denis) est soumis au jugement d'Eustache de Ferroles, définiteur de la province du Saint-Sacrement. On ne trouve plus rien de tel ensuite. C'est que le statut de l'écriture a changé : d'un vecteur d'une identité régulière, celle de la récollection, elle est devenue le support d'une identité provinciale, ce que la liste élaborée par Hyacinthe Le Febvre révèle clairement en 1677. Le soutien de la province dans les entreprises d'écriture et de publication est par ailleurs indispensable. Au moment de l'écriture, d'abord: bien des auteurs avouent à leurs lecteurs avoir écrit à la demande de leur supérieur désireux de voir sur le marché un livre sur le sujet. C'est le cas d'Archange de Clermont à propos des œuvres de piété qui ont lieu au Calvaire de Romans ${ }^{42}$, qui à la fois se propose, et est désigné par son provincial pour écrire un mémorial de ce lieu. Au moment de la publication, ensuite: Jean-Marie L'Escrivain écrit ainsi précocement (1628) dans la préface d'un de ses ouvrages, que sa prolixité est telle, que sa province n'a pas les moyens de financer la publication de la totalité de ses sermons :

\begin{abstract}
"J'aurais voulu donner en quatre volumes d'amples commentaires sur les quatre évangélistes ; mon vœu de pauvreté m'empêche de réaliser ce projet. Nous vivons dans le siècle de l'or. C'est lui qui assure considération ; sans lui rien ne peut se faire. Aussi en suis-je réduit à offrir seulement au public les éclaircissements sur la prophétie de saint Jude que j'ai développés en prêchant l'avent dans la cathédrale de Metz ${ }^{43}$.»
\end{abstract}

L'importance de l'identité provinciale dans le processus d'écriture est confirmée par un autre indice, celui des lieux d'édition (carte 1). Le rayon d'action des écrivains est extrêmement limité. Certes, un livre publié dans une petite ville de province a pu ensuite être vendu et lu ailleurs dans le royaume, voire en Europe, mais des études sur la composition des bibliothèques ecclésiastiques ont bien montré la faible part de l'édition provinciale - et, le plus souvent, purement locale - dans ces collections ${ }^{44}$. Dans l'ensemble, les presses parisiennes ont su attirer des auteurs de toute la France (46\% des ouvrages dus à des récollets sont publiés ou republiés à Paris, quelle que soit la province d'origine), mais le tableau reste surtout celui d'un éparpillement géographique qui correspond aux limites provinciales. Ainsi, dans la province Saint-Bernardin, qui couvre un vaste sud-est s'étendant du Lubéron à Montpellier et son arrière-pays, sur 21 publications dotées d'une adresse typographique, 4 ont été exécutées à Avignon, 2 à Béziers, 2 à Marseille, une à Pézenas, 3 à Paris et 7 à Lyon qui fait figure de pôle régional de l'édition. Les religieux de la province du Saint-Sacrement se font publier

\footnotetext{
${ }^{40}$ H. MONTLOUIS, La Généalogie de l'âme fidèle, deduicte depuis le commencement du monde jusques à nostre temps, par l'espace de 57 siècles, ou autrement l'histoire de l'estat de l'Eglise, tant sous la loy de Nature que tous la Loy Escrite, \& de Grace, Lyon, 1649.

41 I. COTTART, Le manuel du bon religieux par la pratique en abbrégé de la vie régulière menée avec esprit, conformément à celuy des saints docteurs et maistres de la vie spirituelle, 2e éd. Paris, 1661.

42 Archange de Clermont, Le Transport du Mont-Calvaire de Hierusalem en France, par la pieté d'vn Catholique Dauphinois, Lyon, 1638, « au lecteur, sur le dessein du présent livre ».

43 J.-M. L'ESCRIVAIN, Sacra Christi cunabula, utpote a Circumcisione Domini usque ad quadragesimam, in aede Virginis Deiparae post Adventum Rothomagi praedicata, Paris, 1628, préface, trad. par L. DELISLE, Notice sur Jean-Marie L'Escrivain, religieux franciscain du diocèse de Coutances, Saint-Lô, 1903.

44 On en trouvera divers exemples dans B. DOMPNIER, M.-H. FROESCHLÉ-CHOPARD (dir.), Les religieux et leurs livres à l'époque moderne, Clermont-Ferrand, 2000.
} 
principalement à Tulle et à Toulouse ; ceux de Sainte-Marie-Madeleine, certes largement attirés par la capitale du Royaume qui n'est guère éloignée, n'alimentent pas moins le travail des typographes d'Orléans et de La Flèche. Même Rouen, grand centre de l'édition d'Ancien Régime, est curieusement oubliée des stratégies éditoriales des religieux, avec seulement six publications exécutées dans cette ville. Ce tableau pourrait être étendu à toute l'Europe récollette. Les religieux de la province Saint-André de Flandre, même après leur rattachement définitif à la France en 1713 au traité d'Utrecht, continuent de publier essentiellement à Douai, à Lille, à Saint-Omer. Ceux de la province Saint-Joseph de Flandre privilégient Liège ${ }^{45}$. Le religieux écrit donc pour un public immédiat, local, familier des officines des libraires des petites villes, voire des simples boutiques de merciers ou d'épiciers.

Carte 1 : Les villes d'édition des livres publiés par des récollets, selon la province d'origine de l'auteur

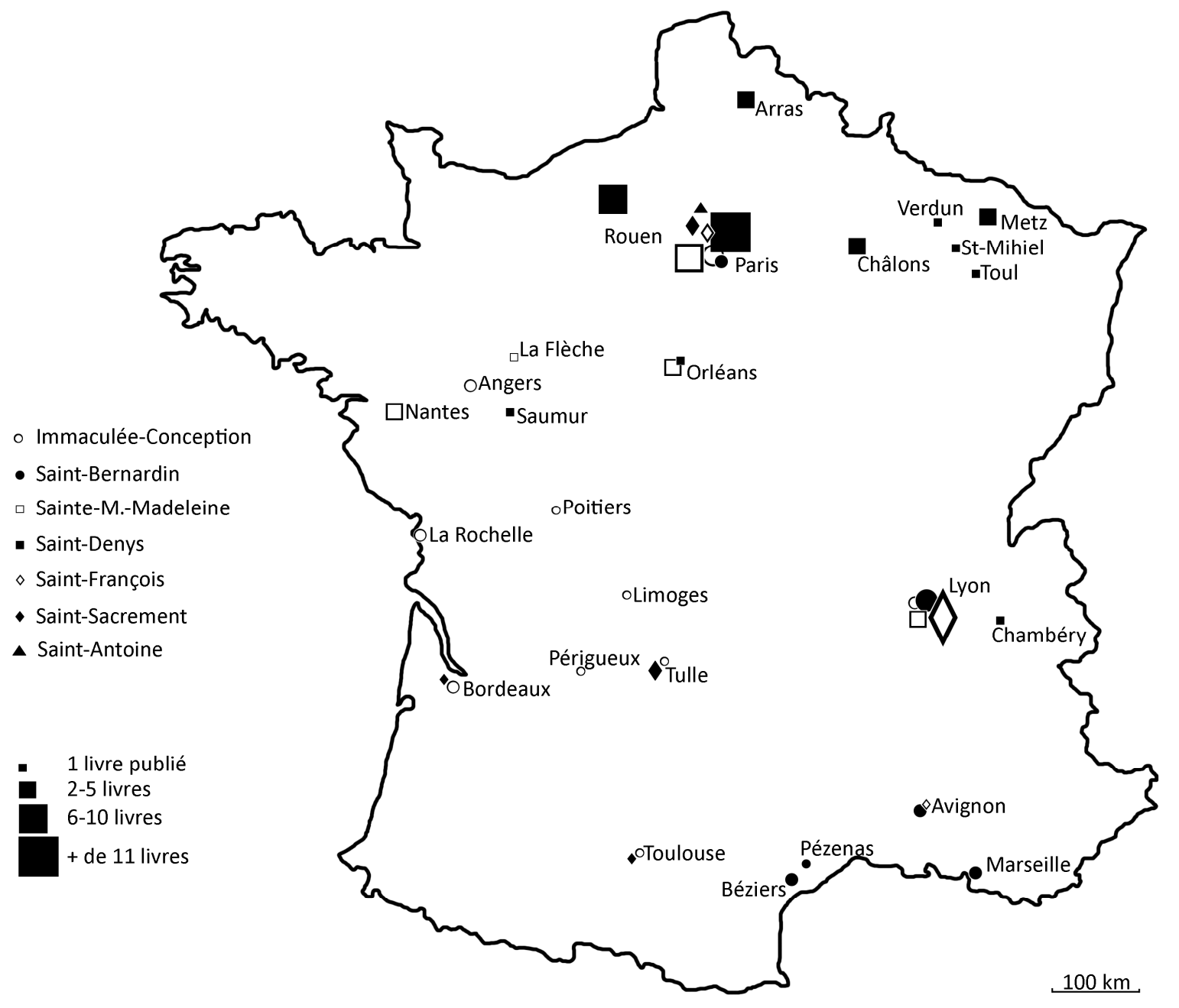

\footnotetext{
${ }^{45}$ S. DIRKXS, Histoire littéraire et bibliographique des Frères Mineurs de l'Observance de St-François en Belgique et dans les Pays-Bas, Anvers, 1885.
} 
En conclusion, l'écrivain récollet du XVII ${ }^{e}$ siècle appartient à une élite très minoritaire de son ordre, avec laquelle il partage une solide formation et un contact permanent avec le monde. Instrumentalisé par sa hiérarchie aux fins de prouver à la société l'efficacité de l'apostolat récollet au moment où la concurrence des ordres est rude, ce personnage est brandi par les premiers historiens de l'ordre comme l'incarnation du bien-fondé de l'installation de l'ordre en France méridionale comme septentrionale. L'écrivain est à la fois la cause et la preuve de l'insertion de la réforme dans le paysage régulier et des bienfaits procurés aux fidèles. C'est aussi de cette manière que les récollets se distinguent dans la famille franciscaine. Paradoxalement, même si les capucins ont produit davantage d'écrivains, ils n'ont pas pensé la fonction de l'auteur avec la même précision, ni préconisé avec autant de vigueur des bibliothèques bien tenues et des études solides, au moins au XVII siècle. La visibilité institutionnelle de l'auteur récollet mérite donc d'être soulignée.

Si l'écrivain agit d'abord de manière empirique, son statut et sa carrière sont peu à peu codifiés ; au milieu du siècle, ils sont stabilisés, même si l'activité en tant que telle ne prend jamais l'autonomie qu'elle a, par exemple, chez les jésuites. La part de l'initiative individuelle est impossible à mesurer mais il est certain qu'elle est largement accompagnée par les autorités, qui incitent, sinon à écrire, du moins à publier.

Pourtant, ce statut n'a pas été promis à une belle prospérité. Tout juste mûri et abouti, au temps d'un Hyacinthe Le Febvre, d'un Séraphin Picot ou, une génération plus tard, d'un Candide Chalippe, le modèle s'essouffle aussitôt et ne suscite plus guère de vocations. Au XVIII siècle, l'on réédite quelques succès des décennies précédentes, comme Zacharie Laselve et son Annus apostolicus (1727, 1760). Dans les années 17601770, Paschase Du Tronc, Hubert Hayer, Fulgence Férot et Constance Miet semblent renouer avec le modèle d'écrivain apostolique qu'avait décrit, un siècle plus tôt, Hyacinthe Le Febvre. Mais ils sont les seuls. Il reste de cet âge d'or que constitue le XVIIe siècle, un vaste réservoir d'auteurs ascétiques où le XIXe siècle puisera longuement, en rééditant Maximien Bernezay, Séverin Rubéric, Charles Jouye, Jacques d’Embrun entre autres. 